Helgoländer wiss. Meeresunters. 32, 305-312 (1979)

\title{
Meiofaunaentwicklung auf künstlichen Weichböden in der Kieler Bucht*
}

\author{
W. SCHEIBEL ${ }^{1} \&$ H. RUMOHR ${ }^{2}$ \\ ${ }^{1}$ Zoologisches Institut der Universität Kiel; \\ Hegewischstr. 3, D-2300 Kiel, \\ und \\ ${ }^{2}$ Institut für Meereskunde der Universität Kiel; \\ Düsternbrooker Weg 20, D-2300 Kiel, Bundesrepublik Deutschland
}

\begin{abstract}
Meiofauna development on artificial soft bottoms in Kiel Bay. The "Benthosgarten", an enclosed area in the western Baltic Sea, is used for benthos ecology experiments. It consists of different kinds of sediment containers filled with "artificial" soft bottom. These containers were sampled by divers over a one-year period to observe the development of the meiofauna population. The first settlement, mainly by nematodes, took place immediately after exposure, and six months later the population was stabilized. The meiofauna consists mainly of nematodes and harpacticoides. Biomass and density are generally lower than in the surrounding area. Only the harpacticoid fauna is well represented by different species and has more individuals per $\mathrm{m}^{2}$ than the surrounding area. Biological aspects of the specific features of the sediment containers, e. g. size and their distance to the sea bottom are discussed.
\end{abstract}

\section{EINLEITUNG}

Im Rahmen der experimentellen in-situ-Untersuchungen zur Bodentierökologie im "Benthosgarten" (Brunswig et al., 1976; Arntz \& Rumohr, 1978; Rumohr, 1978) wurde auch die Initialbesiedlung, Sukzession und Biomasseentwicklung der Meiofauna im Laufe von zwei Jahren ermittelt.

Meiofauna ist hier gemeint als "hard-fauna", die die Gruppen der Nematoden, Harpacticoiden, Ostracoden, Halacariden und Tardigraden einschließt. Die "soft-fauna", wie z. B. Gastrotrichen, Archianneliden, Turbellarien etc., sind in formolfixierten Proben nicht quantitativ erfaßbar und konnten nicht berücksichtigt werden. Die temporären Meiofaunaanteile wie junge Bodenstadien der Mollusken und frühe Polychaeten- und Crustaceenstadien sind Gegenstand einer besonderen Untersuchung (Rumohr, in Vorbereitung). Das heißt, es wurden Nematoden, Harpacticoiden, Ostracoden und - soweit vorhanden - Tardigraden und Halacariden untersucht.

Diese Versuche schließen an frühere Versuche auf "Schwebesubstraten" im gleichen Gebiet an (Sarnthein \& Richter, 1974; Scheibel, 1974). Ziel dieser Arbeit ist nicht die

* Beitrag des Sonderforschungsbereiches 95, Nr. 215 
Erörterung allgemeiner Probleme der Meiofauna im marinen Sublittoral, sondern die notwendige Ergänzung zu Untersuchungen an der Makrofauna, um hieraus ein Gesamtbild der Vorgänge bei der Neubesiedlung von unbewohnten Substraten zu gewinnen. In der Kieler Bucht kommt es am Boden häufig zu Sauerstoffmangel verbunden mit Schwefelwasserstoffbildung (Arntz et al., 1976), wobei die Fauna weitgehend ausgelöscht und der Boden zur Neubesiedlung freigegeben wird. Auch menschliche Einflüsse können ähnliche Zustände hervorrufen, wenn man nur an Sandgewinnung oder an Baggerschüttstellen denkt.

\section{MATERIAL UND METHODE}

Der Versuchsaufbau des "Benthosgarten" (Abb. 1) ist bereits an anderer Stelle dargestellt worden (Arntz \& Rumohr, 1978; Rumohr, 1978). Zum besseren Verständnis soll aber hier eine kurze Beschreibung gegeben werden. Alle Behälter, die im Versuch verwendet wurden, befinden sich in einem Areal von $30 \times 30 \mathrm{~m}$ Ausdehnung. Dieses Areal ist von einem Algen-Fangzaun aus Netzmaterial umgeben ( $1 \mathrm{~m}$ Höhe). Drei Typen von Behältern wurden verwendet: Polyäthylendosen (einen Liter fassend) auf dem Meeresboden und auf Tischen in $70 \mathrm{~cm}$ Höhe; $0,4 \mathrm{~m}^{3}$ fassende Eternitwannen mit $1,5 \mathrm{~m} \varnothing$, deren Oberfläche ca. $40 \mathrm{~cm}$ über der der Umgebung liegt, sowie je drei $0,1 \mathrm{~m}^{3}$ fassende Polyäthylenwannen als Schwebesubstrate, 2 bzw. $5 \mathrm{~m}$ über dem Meeresboden. Diese Wannen befanden sich in luftgefüllten Reifen, die durch Drahtseile mit am Boden liegenden Betonsockeln verbunden waren und schwebend im Wasser gehalten wurden.

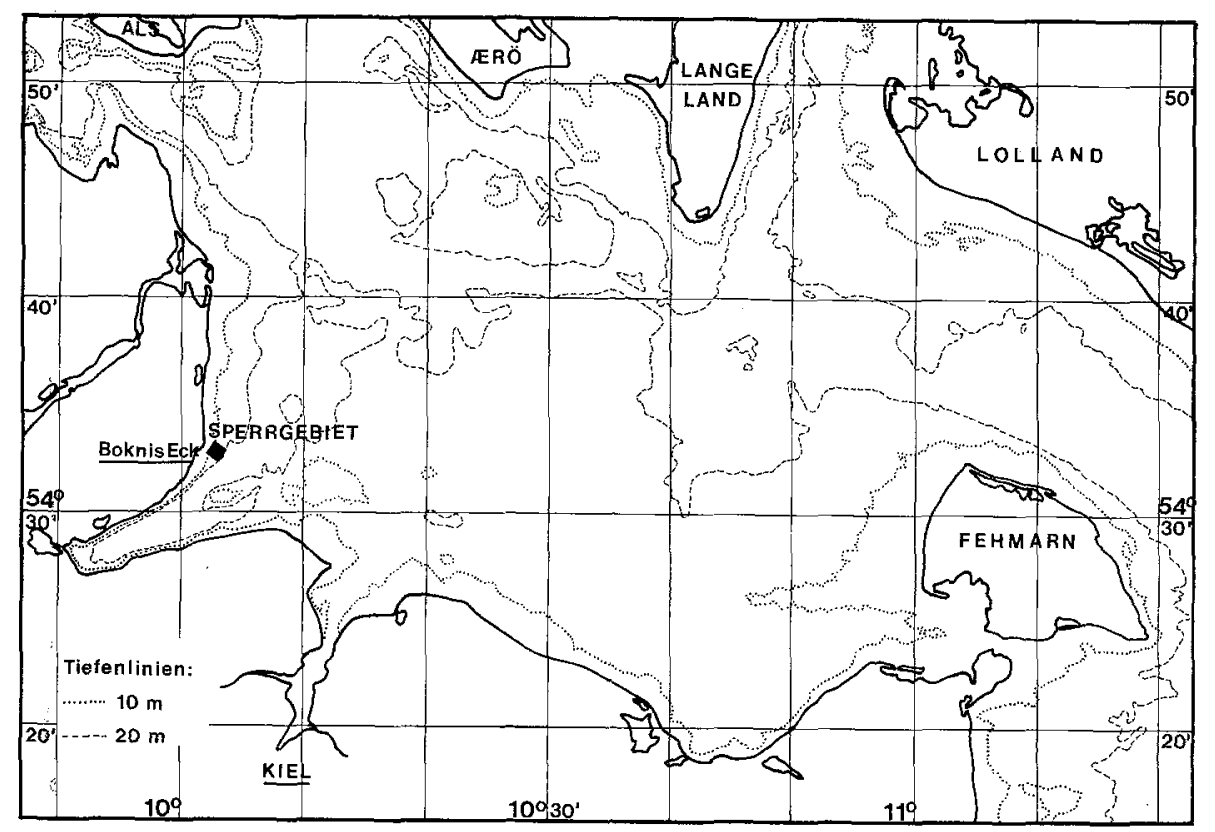

Abb. 1: Lage des Sperrgebietes des SFB 95 (Untersuchungsgebiet) in der Kieler Bucht 
Innerhalb des abgegrenzten Areals befanden sich die Eternitwannen $(1,5 \mathrm{~m} \varnothing)$ auf der einen Seite in zwei Reihen angeordnet; auf der anderen Seite gegenüber die Schwebesubstrate. Im rechten Winkel dazu, auf der dritten Seite, standen die Tische (70 cm hoch) mit Kleinbehältern (einen Liter fassend). Die Kleinbehälter gleichen Inhalts in Meeresbodenhöhe lagen in 2 Doppelreihen in der Mitte des Areals.

Alle Behälter waren mit terrestrischem Substrat gleicher Konsistenz (82\% Feinsand, $\varnothing 125 \mu \mathrm{m}$ und $18 \%$ Ton) gefüllt. Diese Zusammensetzung entspricht dem Meeresboden im Untersuchungsgebiet. Das verwendete Material stammt aus einer Kiesgrube und wurde nicht besonders behandelt.

Alle Substrate wurden zum gleichen Zeitpunkt (Dezember 1975) exponiert. Die Proben wurden bereits nach einer Woche in 14tägigem, später monatlichem Abstand durch Taucher gewonnen. Die hier verwendeten Stechrohre erfaßten $10 \mathrm{~cm}^{2} \hat{=} 50 \mathrm{~cm}^{3}$. Die in $10 \%$ igem Formol fixierten Proben wurden durch Siebe mit 200, 100 und $40 \mu \mathrm{m}$ Maschenweite geschlämmt (Leitungswasser), die Fauna mit Pipetten abgetrennt, gezählt, sortiert und später mit einer Electronic-G 2-Cahnwaage gewogen. Hierbei wurde die Meiofauna auf Whatman-Filter aufgetragen und 24 Stunden im Trockenschrank bei $60^{\circ} \mathrm{C}$ getrocknet. In gleicher Weise wurden jeweils bei jeder Probe 10 Vergleichsfilter behandelt. Alle Filter waren vorher bis zur Gewichtskonstanz getrocknet worden. Die auf den Abbildungen 2 bis 5 und im Text verwendeten Symbole bedeuten: $B=1-1$-Behälter am Boden, $W=0,4-$ $\mathrm{m}^{3}$-Wannen am Boden ( $40 \mathrm{~cm}$ über dem Boden), $\mathrm{T}=1-1-$ Behälter auf Tischen $(70 \mathrm{~cm}$ hoch), $\mathrm{S}_{19}=$ Schwebesubstrate in $19 \mathrm{~m}$ Wassertiefe ( $2 \mathrm{~m}$ über dem Boden), $\mathrm{S}_{16}=$ Schwebesubstrate in $16 \mathrm{~m}$ Wassertiefe ( $5 \mathrm{~m}$ über dem Boden).

\section{ERGEBNISSE}

Die Initialbesiedlung erfolgt auf den Substraten sehr bald nach der Exponierung. Schon am ersten Probenentnahmetermin (eine Woche nach dem Aussetzen) waren etwa 10 Nematoden $/ 10 \mathrm{~cm}^{2}$ anzutreffen. Die weitere Entwicklung für die einzelnen Substrate ist in den Abbildungen 2 und 3 dargestellt. Im Vergleich zum Meeresboden bleibt die Individuenzahl (hauptsächlich Nematoden) auf den künstlichen Substraten aber deutlich niedriger; der ermittelte $\varnothing$-Wert am Boden in $20 \mathrm{~m}$ Tiefe beträgt 1994 Individuen $/ 10 \mathrm{~cm}^{2}$ (Scheibel, 1976). Auf den Versuchsbehältern beträgt die $\varnothing$-Individuenzahl pro $10 \mathrm{~cm}^{2}$ für $S_{16}=202$, $\mathrm{S}_{19}=100, \mathrm{~T}=179, \mathrm{~W}=547$ und $\mathrm{B}=265$. Mit zunehmender Entfernung des Substrates vom Meeresboden ist die durchschnittliche Besiedlungsdichte geringer. Das erscheint auch plausibel, da die Ausbreitungsmöglichkeiten der Meiofauna erschwert sind (Gerlach, 1977). Der niedrige Wert bei B (auf Boden-Niveau) könnte durch den mühelosen Zugriff durch evertebrate Räuber zustande kommen. Außerdem ging hier treibendes Algenmaterial "fegend" darüber hinweg.

Allen Substraten gemeinsam ist eine stetige Zunahme und sich einpendelnde Individuendichte (Abb. 2, 3). Außer Nematoden und Harpacticoiden treten noch Ostracoden, Halacariden und - in einem Einzelfall - Tardigraden auf. Meist sind es nur Einzelexemplare oder doch nur wenige Tiere (Höchstzahl der Ostracoden $=12$ Tiere). Auf die speziellen Merkmale der Harpacticoiden-Fauna wird anschließend noch besonders eingegangen. Die Substrate auf Meeresboden-Niveau zeigen eine schneller einsetzende Stabili- 
sierung der Meiofauna-Assoziationen. Die Probennahme über zwei volle Jahre wurde nur für die Wannen (W) am Boden fortgeführt. In der Besiedelung trat jedoch keine Anderung mehr ein; daher wurde auf eine zusammenhängende graphische Darstellung verzichtet.

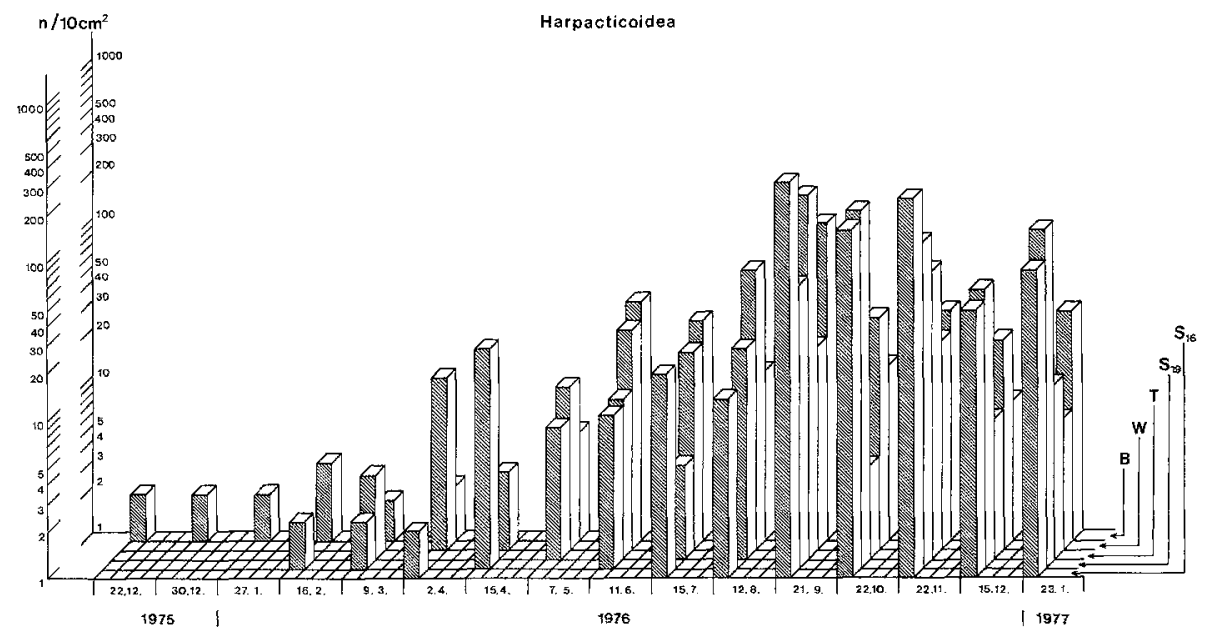

Abb. 2: Individuenzahlen der Harpacticoiden auf allen untersuchten Substraten

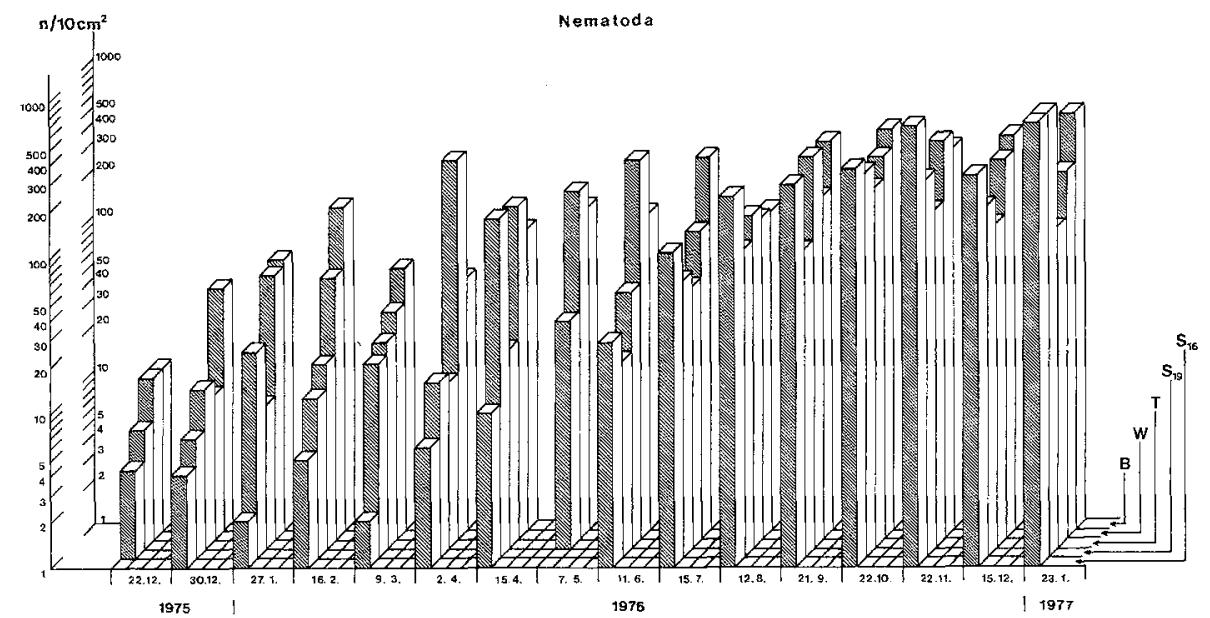

Abb. 3: Individuenzahlen der Nematoden auf allen untersuchten Substraten

\section{Biomasse}

Die Biomasse wurde durch direktes Wägen als Trockengewicht ermittelt. Da dieses Verfahren doch mit einigen Mängeln, vor allem bei sehr niedrigen Individuenzahlen, behaftet ist, werden hier zweckmäßigerweise nur die $\varnothing$-Werte angegeben (Tab. 1). 
Tabelle 1

Durchschnittliche Individuenzahlen und Trockengewicht $\left(\mathrm{mg} / 10 \mathrm{~cm}^{2}\right)$ der Meiofauna auf den verschiedenen Versuchsbehältern in abnehmender Entfernung vom Meeresboden. (Symbolerläuterungen siehe Text)

\begin{tabular}{|cccc|}
\hline $\begin{array}{c}\text { Versuchsbe- } \\
\text { dingungen }\end{array}$ & $\begin{array}{c}\varnothing \text {-Individuen- } \\
\text { zahl } 10 \mathrm{~cm}^{2}\end{array}$ & $\begin{array}{c}\varnothing \text {-Trockengewicht } \\
\left(\mathrm{mg} / 10 \mathrm{~cm}^{2}\right)\end{array}$ & $\begin{array}{c}\text { Entfernung vom } \\
\text { Meeresboden }\end{array}$ \\
\hline $\mathrm{B}$ & 265 & 0,204 & \\
$\mathrm{~W}$ & 547 & 0,105 & $0,1 \mathrm{~m}$ \\
$\mathrm{~T}$ & 179 & 0,120 & $0,4 \mathrm{~m}$ \\
$\mathrm{~S}_{19}$ & 100 & 0,306 & $0,7 \mathrm{~m}$ \\
$\mathrm{~S}_{16}$ & 202 & 0,101 & $2,0 \mathrm{~m}$ \\
\end{tabular}

Tabelle 2

Harpacticoiden-Arten auf den ausgebrachten Substraten

\begin{tabular}{|c|c|c|c|c|c|}
\hline Species & $\mathrm{S}_{19}$ & $\mathrm{~S}_{16}$ & $\mathrm{~T}$ & B & W \\
\hline Longipedia minor T. \& A. Scott & 1 & 4 & - & 2 & - \\
\hline Halectinosoma gotbiceps Giesbrecht & 54 & 77 & 35 & 38 & 42 \\
\hline Hastigerella tenuissima Klie & - & - & 1 & - & - \\
\hline Tachidius discipes Klie & - & - & - & 1 & - \\
\hline Microarthridion littorale (Poppe) & - & - & - & - & 1 \\
\hline Danielssenia typica Boeck & 48 & 16 & 41 & 107 & 169 \\
\hline Harpacticus flexus Brady \& Robertson & - & - & - & 1 & - \\
\hline Parampbiascopsis longirostris Claus & 2 & 4 & 27 & 85 & 82 \\
\hline Amphiascoides debilis Giesbrecht & 13 & 45 & 11 & 19 & 23 \\
\hline Stenbelia palustris Brady & 1 & 1 & 9 & 32 & 8 \\
\hline Amphiascus minutus (Claus) sp. I Lang & - & 4 & - & 4 & 8 \\
\hline Typhlamphiascus typblops (Sars) & - & - & - & - & 1 \\
\hline Ameira parvula Claus & 351 & 349 & 144 & 72 & 97 \\
\hline Ameira divagans Nicholls & - & 16 & 2 & 1 & - \\
\hline Proameira biddensöensis Schäfer & 3 & 18 & - & - & - \\
\hline Nitocra typica Boeck & 4 & 8 & - & 2 & 1 \\
\hline Nitocra spec. & 1 & - & - & - & - \\
\hline Scutellidium spec. & - & - & - & 1 & - \\
\hline Leptopontia curvicauda T. Scott & 1 & - & - & - & - \\
\hline Paraleptastacus spinicauda (T. \& A. Scott) & 1 & - & - & - & - \\
\hline Intermedopsyllus intermedius (") & 1 & - & - & - & - \\
\hline Scottopsyllus minor T. \& A. Scott & 1 & - & - & - & - \\
\hline Mesochra pygmaea Claus & 3 & 23 & 2 & 3 & 10 \\
\hline Mesochra inconspicua T. Scott & 2 & - & 1 & - & 1 \\
\hline Huntemannia jadensis Poppe & - & - & - & 1 & - \\
\hline Enhydrosoma propinquum (Brady) & - & - & - & 4 & 6 \\
\hline Enbydrosoma longifurcatum Sars & - & - & 1 & - & - \\
\hline Laophonte thoracica Boeck & 4 & 46 & 21 & 11 & 29 \\
\hline Laophonte brevispinosa (Sars) & - & - & - & 14 & 16 \\
\hline Heterelaophonte minuta Boeck & - & - & 1 & - & 2 \\
\hline Asellopsis intermedia (T. Scott) & - & - & - & - & 2 \\
\hline Onychocamptus brevispinosus (Sars) & - & - & - & - & 2 \\
\hline Paronychocamptus curticaudatus (Boeck) & - & - & 1 & 3 & 10 \\
\hline
\end{tabular}


Die $\varnothing$-Werte bleiben auch hier, entsprechend der Individuenzahl, deutlich hinter denen des Meeresbodens in $20 \mathrm{~m}$ Tiefe zurück, wo $560 \mathrm{mg}$ Trockengewicht $/ \mathrm{m}^{2}$ erreicht werden (Scheibel, 1976). Aufgrund der unterschiedlichen Individuengröße bringt das $\varnothing$ Gewicht einer Probe nicht immer die $\varnothing$-Individuenzahl zum Ausdruck.

\section{Merkmale der Harpacticoiden-Fauna}

Die Individuenzahl bleibt auf allen Substraten hinter der der Nematoden zurück. Gleiches gilt im wesentlichen für die Initialbesiedlung; sie setzt zögernder ein, stabilisiert sich aber mit zunehmender Dauer des Experimentes, ähnlich wie die Nematodenfauna.

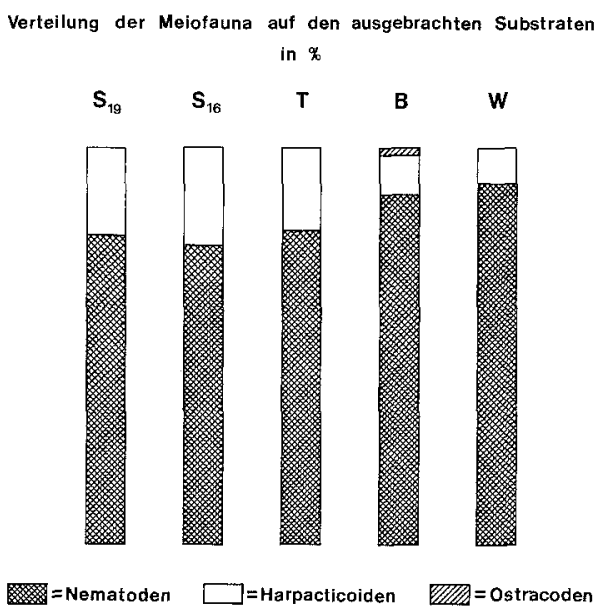

Abb. 4: Prozentuale Verteilung der Meiofauna auf den ausgebrachten Substraten

Wie schon in früheren Experimenten mit Schwebesubstraten (Scheibel, 1974) ermittelt wurde, dominieren aber auch diesmal nur wenige Arten. Die Dominanz der sechs häufigsten Arten ist in Abbildung 5 für alle Substrate dargestellt. Als weiteres Charakteristikum der Fauna auf den ausgebrachten Substraten gegenüber dem Meeresboden muß erwähnt werden, daß die Artenzahl auf den ausgesetzten Substraten wesentlich höher ist, wenn auch ein großer Teil der Tiere nur als Einzelexemplar vorliegt (siehe Tabelle 2). In $20 \mathrm{~m}$ Tiefe wurden früher 5 Arten gefunden (Scheibel, 1976), jetzt 11, auf den ausgebrachten Substraten dagegen 33! Das Artenspektrum ist viel heterogener als auf dem umgebenden Meeresboden. Es vereinigen sich Faunenelemente des reinen Sandbodens mit denen des schlickigen Feinsandes. Wie schon früher erwähnt (Scheibel, 1974), sind es aber wieder besonders zwei Arten, die stark dominieren, nämlich Ameira parvula und Danielssenia typica. Von diesen zeigt Ameira parvula die geringste Substratpräferenz und stellt den anderen Arten gegenüber die höchste Individuenzahl, besonders auf den Schwebesubstraten. 
Dominanz der häufigsten Harpacticoiden-Arten im "Benthosgarten"
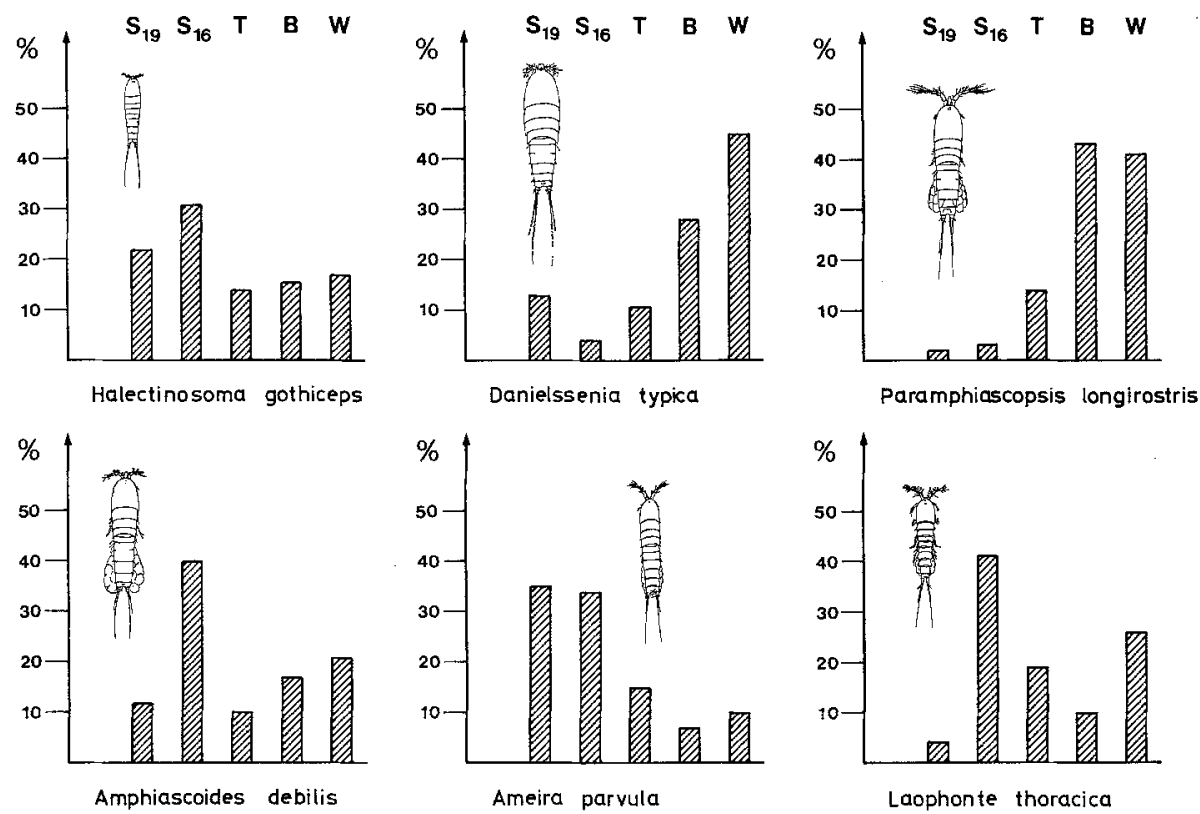

Abb. 5: Dominanz der sechs häufigsten Harpacticoiden-Arten auf den ausgebrachten Substraten

\section{DISKUSSION}

Die Ergebnisse zeigen bezüglich der Meiofauna einen Verlauf, der mehr oder weniger erwartet werden konnte; die Besiedlung deckt sich im wesentlichen mit derjenigen des früheren Versuches mit Schwebesubstraten (Sarnthein \& Richter, 1974; Scheibel, 1974). Die Individuenzahlen und das Verhältnis Nematoden/Copepoden sind vergleichbar. Es bleibt die Frage, warum die Initialbesiedlung mit zunehmender Entfernung der Substrate vom Meeresboden zögernder einsetzt und ebenso auch die Stabilisierung der MeiofaunaAssoziationen. Die später einsetzende Initialbesiedlung auf den Schwebesubstraten hängt sicher mit der exponierteren Lage und damit erschwerten Ausbreitungsmöglichkeiten der Meiofauna zusammen. Über die Möglichkeiten der Verbreitung von Meiofauna ist von Gerlach (1977) eine zusammenfassende Darstellung gegeben worden. Zumindest für die Schwebesubstrate gilt, daß die Halteseile als "Brücke" für die Meiofauna dienen können; dies wurde im Experiment (1974) geprüft. Hinzu kommen die Wasserbewegung und treibende Partikel verschiedener Größe als Transportmittel.

Die frühere Stabilisierung der Sukzession am Boden hängt sicher auch mit dem Nahrungsangebot zusammen, da hier ein besserer Kontakt zum umgebenden Meeresboden besteht. Doch stehen gerade auf diesem Gebiet der trophischen Beziehungen noch viele Probleme zur Klärung an, so daß eine abschließende Wertung hier nicht gegeben werden kann. 


\section{ZITIERTE LITERATUR}

Arntz, W. E., Brunswig, D. \& Sarnthein, M., 1976. Zonierung von Mollusken und Schill im Rinnensystem der Kieler Bucht (westliche Ostsee). Senckenberg. marit. 8, 189-269.

- \& Rumohr, H., 1978. The "Benthosgarten": Field experiments on benthic colonization in the western Baltic. II. Subsequent successional stages. Kieler Meeresforsch. (Im Druck).

Brunswig, D., Arntz, W. E. \& Rumohr, H., 1976. A tentative field experiment on population dynamics of macrobenthos in the western Baltic. Kieler Meeresforsch. 3, 49-59.

Gerlach, S. A., 1977. Means of meiofauna dispersal. Mikrofauna des Meeresbodens 61, 89-103.

Lang, K., 1948. Monographie der Harpacticiden. Ohlsson, Lund, 1-2, 1-1682.

Rumohr, H., 1978. The "Benthosgarten": Field experiments on benthic colonization in the western Baltic. I. Initial colonization. Kieler Meeresforsch. (Im Druck).

Sarnthein, M. \& Richter, W., 1974. Submarine experiments on benthic colonization of sediments in the western Baltic Sea. I. Technical layout. Mar. Biol. 28, 159-164.

Scheibel, W., 1974. Submarine experiments on benthic colonization of sediments in the western Baltic Sea. II. Meiofauna. Mar. Biol. 28, 165-168.

- 1976. Quantitative Untersuchungen am Meiobenthos eines Profils unterschiedlicher Sedimente in der westlichen Ostsee. Helgoländer wiss. Meeresunters. 28, 31-42. 